\title{
El modelo de aprendizaje TPACK y su impacto en la innovación educativa desde un análisis bibliométrico
}

\section{The TPACK learning model and its impact on educational innovation from a bibliometric analysis}

\author{
Carlos Darío Vásconez Paredes \\ Universidad Politécnica Salesiana, Quito, Ecuador \\ cvasconez@ups.edu.ec \\ https://orcid.org/0000-0002-1574-346X \\ Esteban Mauricio Inga Ortega \\ Universidad Politécnica Salesiana, Quito, Ecuador \\ einga@ups.edu.ec \\ https://orcid.org/0000-0002-0837-0642
}

Recepción: 01/06/2021 | Aceptación: 17/08/2021 | Publicación: 10/09/2021

Cómo citar (APA, séptima edición):

Vásconez Paredes, C.D., y Inga Ortega, E.M. (2021). El modelo de aprendizaje TPACK y su impacto en la innovación educativa desde un análisis bibliométrico. INNOVA Research Journal, 6(3), 79-97. https://doi.org/10.33890/innova.v6.n3.2021.1773

\section{Resumen}

El TPACK sintetiza los conocimientos que se espera que el docente deba tener a la hora del proceso de enseñanza-aprendizaje. Esta investigación contextualiza el Conocimiento del Contenido Pedagógico Tecnológico (TPACK), a la vez que presenta una revisión y un análisis sistemático de la bibliografía hallada a nivel regional y mundial en las bases de datos Web of Science y Scopus entre los años 2016 y 2020, en el cuál hemos considerado los criterios de mayor número de citas y artículos más relevantes dentro de la búsqueda general con la palabra clave TPACK. De igual forma hemos nutrido este análisis con datos estadísticos provenientes de la Organización para la Cooperación Económica y el Desarrollo (OECD). Los métodos usados para la investigación han sido el método Histórico-Descriptivo y el método Analítico-Sintético. En consecuencia, como fruto de este hemos identificado los problemas o variables más comunes dentro de la aplicación del modelo del TPACK y las (posibles) soluciones o consideraciones planteadas desde las 
investigaciones con las que se alimenta este estudio lo que constituye un punto de partida para analizar la implementación de TPACK.

Palabras claves: nuevas tecnologías; desarrollo profesional; tecnología de la educación; tecnologías de la información y de la comunicación; perfeccionamiento de profesores.

\begin{abstract}
TPACK synthesizes the knowledge that teachers are expected to have during the teaching-learning process. This research contextualizes Technological Pedagogical Content Knowledge (TPACK) while presenting a systematic review and analysis of the literature found at the regional and global level in the Web of Science and Scopus databases between 2016 and 2020, in which we have considered the criteria of the highest number of citations and most relevant articles within the general search with the keyword TPACK. We have also nourished this analysis with statistical data from the Organization for Economic Co-operation and Development (OECD). The methods used for the research were the Historical-Descriptive method and the Analytical-Synthetic method. Consequently, as a result, we have identified the most common problems or variables within the application of the TPACK model and the (possible) solutions or considerations raised from the research that feeds this study, which constitutes a starting point for analyzing the implementation of TPACK.
\end{abstract}

Keywords: new technologies; profesional development; educational technology; information and communication technologies; teacher training.

\title{
Introducción
}

La tecnología ha ido creciendo de forma exponencial; en consecuencia, ha entrado de forma autoritaria a las instituciones educativas, y que de alguna forma nos ha encontrado desprecavidos. Esta incursión trae consigo necesidades para la Innovación Educativa, siendo una de ellas la integración de la pedagogía y la tecnología por medio de las Tecnologías de la Información y Comunicación (TIC). Sin embargo, esta integración requiere que los docentes adquieran y desarrollen conocimientos de los contenidos, la pedagogía y tecnología, lo que para Mishra y Koehler (2006) vendría a ser el Conocimiento del Contenido Pedagógico y Tecnológico (TPACK).

En 2020, a raíz de la pandemia por COVID-19, los docentes se vieron obligados a migrar sus clases al entrono online, en el cual se logró palpar las dificultades y desconocimiento tecnológico y pedagógico de los docentes. Si antes de la pandemia ya costaba usar un proyector. Ahora, el desarrollar competencias digitales para usar un Moodle o un software de videoconferencias o cualquier otra TIC que permita la interacción con los estudiantes para favorecer los aprendizajes, resulta un camino duro de roer. La integración de las TIC en el ambiente educativo se venía dando con antelación, sin embargo, debido a la pandemia, esta transición se dio de forma apresurada y obligatoria, haciendo que a muchos docentes les coja por sorpresa; es decir, se esperó hasta verse obligados para incluir las TIC en la educación (Schildkamp et al., 2020).

Por ello, hablar de la implementación del TPACK hoy en día se vuelve cada vez una necesidad a la que no podemos postergar. Hemos sido arrasados por la tecnología y han sido precisamente los estudiantes quienes han llevado esa tecnología a los centros educativos. El uso de tecnologías emergentes como los teléfonos celulares (M-learning) han supuesto un acceso 
inmediato a la información debido a la portabilidad, la inmediatez, conectividad, ubicuidad y adaptabilidad (Ortega-Sánchez y Gómez-Trigueros, 2019). Sin embargo, el uso de TIC continua siendo una excepción, más no una normativa (Chai et al., 2010) que seguramente causa incertidumbre en el retorno a la prespecialidad.

Así pues, el TPACK se vuelve una necesidad para la mejora de la calidad educativa, sin embargo, la aplicación del mismo y consecuentemente la integración de las TIC se vuelve un reto cada vez mayor, pues no solo depende el conocimiento de los docentes, sino también de los contextos en los que estén (Cheng, 2017; Ling Koh et al., 2014), el acceso a las tecnologías, las condiciones demográficas, los temores y creencias, el tiempo de dedicación para la preparación de la clase, así como la capacitación permanente y actualizada, pues -podría decirse- que las TIC tienen caducidad, pues el conocimiento tecnológico rápidamente se vuelve obsoleto haciendo que la adquisición de nuevos conocimientos y sus aplicaciones resulten en una continua transformación en entornos culturales y sociales (Angeli y Valanides, 2009). Por lo tanto, la implementación del TPACK, no se reduce a una mera decisión política-educativa, sino que además debe responder a las características particulares de lugar en donde se desee aplicar. Así mismo, la formación de los docentes en TIC no se resumen a una formación generalista y universal, sino que esta debe ir de la mano de las necesidades específicas de cada docente, del área y de la asignatura que imparta (Chai et al., 2010).

El estudio busca contextualizar mediante un análisis bibliométrico las experiencias a la hora de implementar el TPACK, para lo cual hemos emprendido una exhaustiva investigación de artículos en las bases de datos más importantes a nivel mundial. De esta forma, aspiramos constituir un punto de partida para la investigación y la ejecución exitosa de TPACK, es decir, saber a ciencia cierta lo que debemos considerar a la hora de aplicar TPACK. Así mismo, este trabajo es uno de los primeros estudios bibliométricos hechos en Ecuador que analiza, estudia y contextualiza el TPACK según experiencias a nivel mundial y sin duda permitirá abordar y desarrollar el tema con mayor claridad.

En la figura 1, mostramos los elementos o variables para tener en cuenta a la hora de la aplicación del TPACK. 


\section{Figura 1}

Gráfico conceptual TPACK: Integración y análisis de las variables a considerar antes de implementar

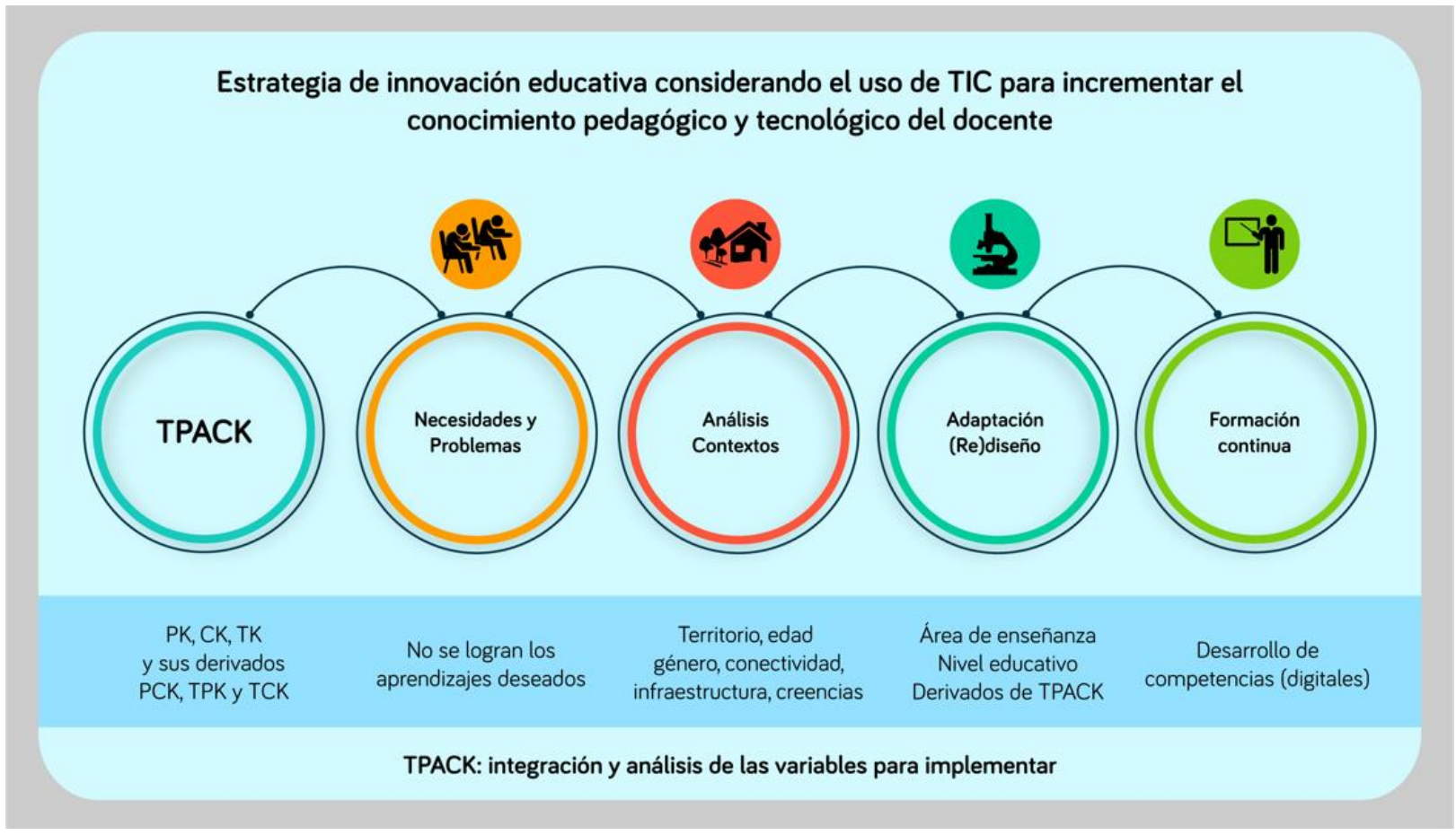

\section{Trabajos Relacionados}

Trabajos presentados por Shulman $(1986,1987)$ plantean las bases de lo que hoy conocemos como TPACK. Él propone que los conocimientos pedagógicos es una dimensión que debe ir a la par con el conocimiento de los contenidos (currículo), es decir, que a más de conocer lo que se enseña, se debe saber cómo enseñar. Por lo que, ser capaz de enseñar una asignatura es tan importante como tener el conocimiento de la misma (Ekiz-Kiran y Boz, 2020). En consecuencia, Shulman propone el Conocimiento de Contenido Pedagógico (PCK) como resultado de la intersección de Conocimiento del Contenido (CK) y el Conocimiento Pedagógico (PK), pues anteriormente se veía a cada conocimiento como dos instancias diferentes y separadas. Shulman (1986) propone juntar estas instancias. Es así que el PCK ocurre solo cuando el profesor interpreta la asignatura y conoce de las herramientas para compartirla a sus estudiantes (Mishra y Koehler, 2006). Mishra y Koehler (2006) toman las ideas de Shulman, pero además, incorporan a las TIC como un tercer elemento que es transversal.

Para entender lo que nos propone Mishra y Koehler (2006) debemos hacer un desglose de su modelo TPACK. Partimos de la idea que nos propone Shulman, como hemos visto anteriormente el PK y el CK se entrelazan, sin embargo, el Conocimiento Tecnológico (TK) queda de lado, seguramente, porque para la década de los 80 recién la tecnología empezaba a democratizarse con las computadoras y el internet. Es así como años más tarde la tecnología 
vendría a tomar un papel fundamental en el quehacer educativo. Mishra y Koehler plantean no solo analizar de forma aislada el Conocimiento del Contenido (CK), la Pedagogía (PK) y la Tecnología (TK), sino que además proponen analizar en pares. La intercesión de estos 3 derivan en 3 grandes constructos (Cheng, 2017). El Conocimiento del Contenido Pedagógico (PCK), Conocimiento del Contenido Tecnológico (TCK) y el Conocimiento Pedagógico Tecnológico (TPK); y, a su vez, la intercesión de estos 3 grupos da paso al Conocimiento de Contenido Pedagógico y Tecnológico (TPACK). En la tabla 1, se logra identificar las características de cada una de las dimensiones de TPACK.

\section{Tabla 1}

Descripción de las construcciones en el marco TPACK

\begin{tabular}{|c|c|c|}
\hline Sigla & Significado & Descripción \\
\hline$C K$ & Conocimiento del contenido & $\begin{array}{l}\text { Refiere al conocimiento del contenido de la } \\
\text { asignatura }\end{array}$ \\
\hline$P K$ & Conocimiento pedagógico & $\begin{array}{l}\text { Refiere al conocimiento de estrategias para } \\
\text { enseñar }\end{array}$ \\
\hline$T K$ & Conocimiento tecnológico & $\begin{array}{l}\text { Refiere al conocimiento de cómo usar las } \\
\text { herramientas tecnológicas }\end{array}$ \\
\hline$P C K$ & $\begin{array}{l}\text { Conocimiento del Contenido } \\
\text { Pedagógico }\end{array}$ & $\begin{array}{l}\text { Refiere al conocimiento de uso de estrategias } \\
\text { apropiadas para enseñar }\end{array}$ \\
\hline$T P K$ & $\begin{array}{l}\text { Conocimiento Pedagógico } \\
\text { Tecnológico }\end{array}$ & $\begin{array}{l}\text { Refiere al conocimiento de la aplicación de la } \\
\text { tecnología para implementar estrategias para } \\
\text { enseñar }\end{array}$ \\
\hline$T C K$ & $\begin{array}{l}\text { Conocimiento del Contenido } \\
\text { Tecnológico }\end{array}$ & $\begin{array}{l}\text { Refiere al conocimiento para representar el } \\
\text { contenido de la asignatura mediante la tecnología }\end{array}$ \\
\hline TPACK & $\begin{array}{l}\text { Conocimiento del Contenido } \\
\text { Pedagógico y Tecnológico }\end{array}$ & $\begin{array}{l}\text { Refiere al conocimiento para facilitar el } \\
\text { aprendizaje de un contenido específico a través de } \\
\text { una pedagogía y adecuadas tecnologías }\end{array}$ \\
\hline
\end{tabular}

Fuente: Mishra y Koehler (2006).

A partir de las ideas propuestas por Mishra, Koehler, Shulman y Thompson (2006; 1986; 2007) ya se logra identificar al uso de TIC e integración adecuada y contextualizada con los otros contenidos cuenta para lograr una buena calidad educativa o a su vez, un buen proceso de enseñanza-aprendizaje. En consecuencia, las universidades, escuelas y colegios han sido impulsados a invertir en tecnologías. Sin embargo, en esa primicia surge una idea equivoca del uso e implementación de la tecnología como una herramienta que mejorará los resultados educativos o que a su vez resulta ser un símil de innovación, sin embargo, esto no siempre es así; pues la efectividad de la tecnología solo es posible cuando se alinea con el contenido de las asignaturas y su pedagogía (Bostancioğlu y Handley, 2018), es decir, la implementación de la tecnología no significa una mejor calidad educativa como erróneamente se cree.

No obstante, a pesar de esto, una gran mayoría de los docentes han implementado la tecnología en sus clases y en muchos casos sin mayores cambios. Vemos como PowerPoint, YouTube, etc., han sustituido a los retroproyectores y DVD (Sutton y DeSantis, 2017), pero este 
sustitución queda en lo estético; es decir, solo se modernizó la herramienta para acceder a cierto tipo de material o a su vez nos quedamos en el uso de TIC para la (re)transmisión de información y/o contenidos (Koh et al., 2017), principalmente como un instrumento de apoyo para las clases magistrales, exposiciones orales o recursos para ampliar información (Cenich et al., 2019). Así pues, se ha generado la idea errónea que la tecnología es solo un dispositivo y no herramientas e instrumentos que sirven para resolver problemas (Voogt et al., 2013), facilitar contenidos y poder interactuar con los mismos. En ese sentido desde el modelo SAMR, vemos que el uso de TIC apenas se queda en las dos primeras fases, es decir en sustituir y aumentar, habiendo un largo camino para llegar a la fase de aumentar y redefinir.

Sin duda, la implementación de las TIC trae consigo un sinnúmero de dudas y miedos, sobre todo en docentes que han vivido una época ajena a la era tecnológica. Ahora, la implementación de la TIC en el contexto educativo no se reduce a la proyección de un PowerPoint, sino más bien a las formas y los momentos en el que se debe usar (TK y TCK). No se trata de incluir las TIC de forma azarosa (Thompson y Mishra, 2007). Es por ello que no simplemente se debe hablar de la capacitación de los docentes en TIC, sino de una capacitación en competencias digitales, es decir, que los docentes no solo sepan usar TIC, sino que además sepan seleccionar, adaptar, modificar y redefinir TIC para su contexto educativo (Bostancioğlu \& Handley, 2018). En este sentido, las TIC debe implicar un aprendizaje activo en el que se puede y debe experimentar y manipular. Además, debe propiciar la búsqueda de significados, monitorear logros, participar en la resolución de problemas y fomentar el trabajo colaborativo. Por lo tanto, su uso significa el desarrollo de competencias cognitivas, metacognitivas y tecnológicas (Koh et al., 2017) para esto, los docentes deben hacerse de varios enfoques pedagógicos para aprovechar las TIC (Voogt et al., 2013) siendo este, uno de los aspectos a considerarse en los docentes en formación, el énfasis en las habilidades tecnológicas y pedagógicas para enfrentar los desafíos de los sistemas educativos (Valtonen et al., 2017).

En este sentido, la implementación del TPACK se vuelve tan necesario que en cierto punto es inevitable. Sin embargo, para su implementación será necesario tomar en cuenta algunos elementos. Para Cheng, Getenet, Xu et al. (2017; 2017; 2018) las condiciones demográficas, la edad, el género, la experiencia docente, la infraestructura, el tiempo y la naturaleza de los estudiantes e incluso las creencias de los docentes son variables a tener en cuenta a la hora de implementar el TPACK, pues determinan los contextos para una cercanía entre cada uno de los conocimientos (PK, CK, TK). Por ejemplo, las maestras tienen menor confianza a la hora de aplicar la tecnología (TK), pero establecen una mayor confianza cuando se trata del PK, a diferencia de los hombres (Cheng, 2017). No así cuando se trata del PK, ahí las profesoras muestra un mayor dominio que los profesores (Cabero y Barroso, 2016; Cheng, 2017). En un estudio rescatado por Cabero y Barroso (2016) se evidencia que los profesores de matemáticas, tenían un más alto conocimiento en TPACK que las profesoras. Por otro lado, estudios también han hallado que docentes con altos niveles de confianza en sus habilidades tecnológicas, hacen mayor uso de la tecnología en el aula (Chai et al., 2010). Así mismo, hay que considerar las creencias de los docentes en relación a la tecnología y la pedagogía, si bien es cierto, algunos profesores pueden tener el conocimiento y las habilidades para usar TIC, en la práctica terminan por no usarla (EkizKiran y Boz, 2020; Voogt et al., 2013). Esto pues según explica Depaepe (2020) una cosa es disponer de los conocimientos y desear aplicarlos, pero otra cosa ya es ponerlo en acción en el aula.

Esta obra se comparte bajo la licencia Creative Common Atribución-No Comercial 4.0 International (CC BY-NC 4.0) Revista de la Universidad Internacional del Ecuador. URL: https://www.uide.edu.ec/ 
Las condiciones demográficas también requieren de nuestra atención. En un estudio realizado por $\mathrm{Xu}$ et al. (2018) se evidencia la brecha entre los docentes que se encuentran en entornos urbanos y rurales. Aquí bien valdría aclarar que además de la ubicación o proveniencia geográfica vale considerar que los docentes que están en formación presentan mayores dificultes en cuanto a los conocimientos y el acceso a dispositivos tecnológicos. Además, las universidades, dice Xu et al., (2018) deberían proporcionar las condiciones y los recursos para el aprendizaje de TPACK en los profesores en formación con el fin de que se pueda compensar las deficiencias y a la vez disponer de mejores condiciones para el aprendizaje. Así mismo, hay que recalcar que los docentes en formación adquieren un mayor nivel de habilidades tecnológicas y que a su vez están dispuestos a usar la TIC en las aulas. También, poseen una mayor autonomía y autoeficiencia a la hora de integrar TIC en el diseño e implementación del curso (Chai et al., 2010), pero como hemos visto antes, puede resultar una experiencia difícil a la hora de implementar TIC en el aula.

Por esto, existe una creciente necesidad y a la vez, urgencia para que el currículo o plan de estudios esté en conjunción con las TIC (König et al., 2020), pues a diferencia de lo que se cree que la generación milennial y centennial son sinónimos de nativos digitales y consecuentemente expertos en el uso de tecnologías. Esta etiqueta ya ha sido cuestionada por muchos expertos (Tondeur et al., 2011) ya que crecer con tecnologías no significa que sepan usarlas de forma adecuada y sistematizada. En ese sentido, es menester que las TIC se encuentren dentro del currículo para alfabetizar a las generaciones en el uso de TIC.

Así mismo, se evidenció que los docentes desconocían como asociar la pedagogía con la tecnología (Rap et al., 2020) pues bien, muchos de ellos han usado herramientas tecnológicas, se queda en aplicaciones básicas. Es por ello que proporcionar una variedad de herramientas tecnológicas especializadas en el área del conocimiento de los profesores se vuelve categórico para lograr competencias digitales (Canbazoglu Bilici et al., 2016). Sin embargo, se ha visto que los métodos tradicionales de capacitación como talleres o cursos no son adecuados para una comprensión profunda para que los docentes puedan convertirse en usuarios de la tecnología pero aplicada en la pedagogía (Koehler et al., 2007). Así pues, una de las necesidades durante la pandemia fue encontrar contenidos que disponga de un buen diseño, de tal manera que se adapte a las necesidades del estudiante (Mohamad Nasri et al., 2020).

Ya en el plano local, en Ecuador los resultados de TPACK han encontrado en un nivel aceptable para TK, sin embargo, cuando se trata de TPK Sumba Nacipucha, N., Cueva Estrada, J. M., Conde Lorenzo, E., y Mármol Castillo, M. (2020) dan cuenta que la aplicación de TIC podría verse limitada para la presentación de diapositivas, es decir, la educación se presenta de forma unidireccional: docente-estudiante, lo que reafirma la posición de Depaepe (2020), saber usar tecnología no significa que saber aplicarla. Por otro lado, Peña, F. L. M., Peña, F. E. M., \& Sánchez, J. D. A. (2017) en un estudio realizado a docentes y estudiantes, reflejan altos niveles en los conocimientos de TPACK, sin embargo, señalan que los docentes aún deben financiarse su formación en tecnologías educativas y consecuentemente en el desarrollo de competencias digitales.

Ahora bien, en este momento habría que considerar adaptaciones que surgen del modelo TPACK. Por ejemplo, el TLPACK es una de las que llama nuestra atención. Esta adaptación del modelo TPACK, lleva consigo 5 constructos en total. Incorpora el conocimiento del contexto y el 
conocimiento del alumno (Hsu y Chen, 2019). También se ha encontrado otras derivaciones de TPACK, lo que nos pone en evidencia la flexibilidad de este, para responder a las necesidades. A la par también se ha encontrado la educación STEM o su acrónimo en español: Ciencia, Tecnología, Ingeniería y Matemáticas (CTIM). En este sentido, podría decirse que muchos no solo están usando las TIC, sino que además se han lanzado a diseñar sus propios contenidos para la enseñanza, por consiguiente, el TPACK no debería limitarse al uso de las TIC (Ku et al., 2020), sino también al diseño de las mismas.

\section{Metodología}

Para la investigación se consideró hacerla en dos momentos. En un primer momento hemos usado el método histórico-descriptivo en donde caracterizamos el escenario a nivel regional y mundial. Así pues, para esta caracterización se ha prestado atención a 60 artículos científicos, mismos que fueron el resultado de una búsqueda en las bases de datos Web of Science y Scopus entre los años 2016 y 2020. Con el fin de encontrar generalidades se usó como término de búsqueda TPACK y Technological Pedagogical Content Knowledge. En la selección de resultados se filtró a los artículos por su relevancia, número de citas y que al menos cumpla con una de las palabras clave en el campo de búsqueda. A partir de ahí, se realizó una matriz en una hoja de cálculo y de esta forma se logró extraer e identificar con facilidad la temática, formulación del problema y la solución propuesta.

\section{Figura 2}

Diagrama de flujo de la metodología usada

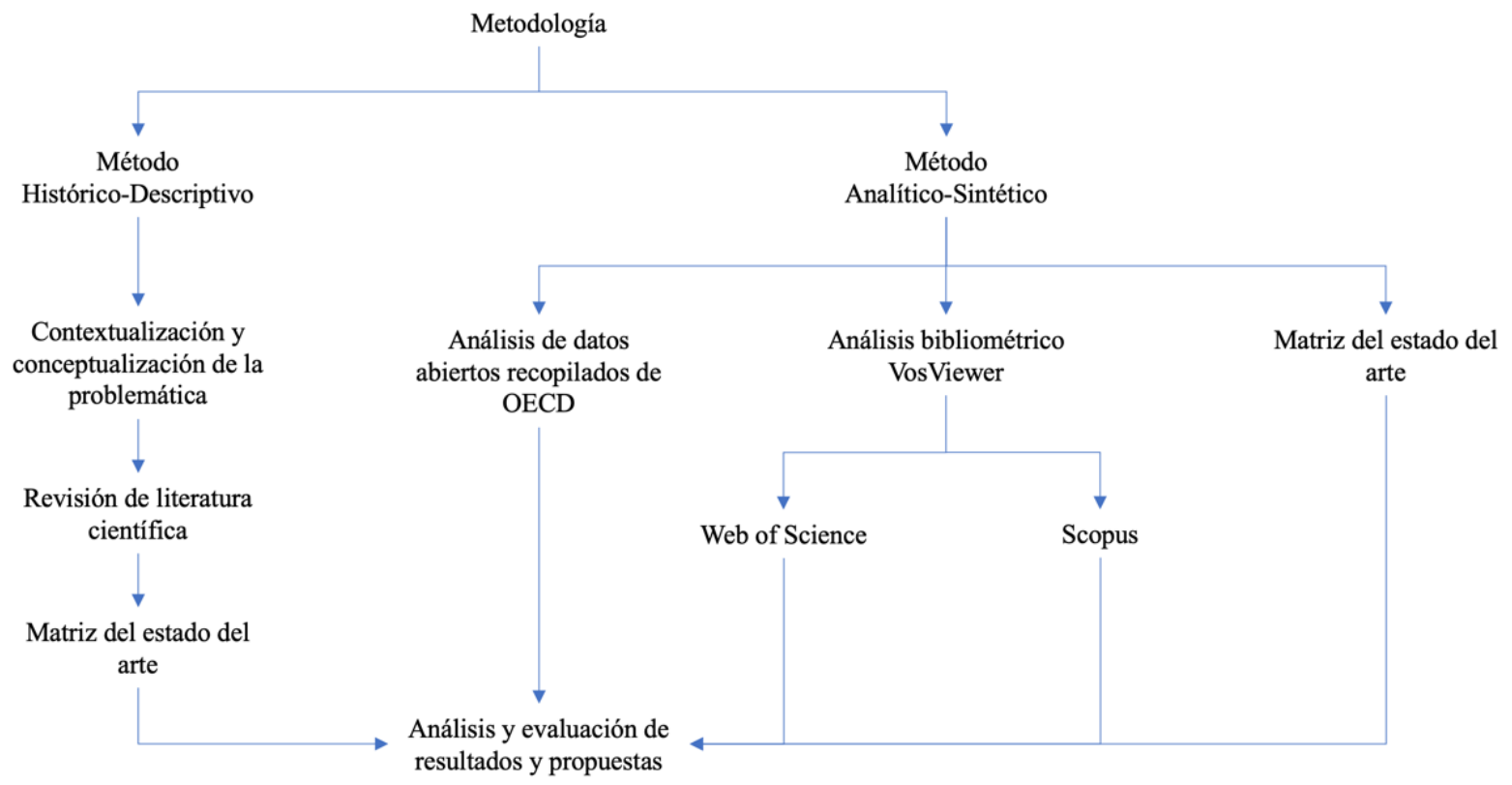

En un segundo momento usamos el método Analítico-Sintético, en el cual vimos pertinente partir de un estudio bibliométrico de la base de datos Web of Science en el que analizamos 2000 artículos científicos y, valiéndonos de VosViewer hemos encontrado relaciones entre países y las 
instituciones que están abordando la temática planteada. Así mismo, se ha logrado identificar a los autores más relevantes en los últimos 4 años. En este segundo momento de igual forma nos hemos valido de la matriz realizada con anterioridad, en la que nos centramos particularmente en 60 artículos y como resultado de estos hemos elaborado gráficas que permiten clarificar nuestro análisis. Además, valiéndonos de datos abiertos provenientes de OECD, se ha logrado evidenciar en cifras como anda la región en términos de acceso a un ordenador desde el hogar, el acceso a internet y el acceso y uso de TIC por hogar y por persona.

Vale mencionar que el estudio, no se centra en un campo exclusivo del conocimiento, es por esto por lo que los artículos analizados provienen de distintas experiencias de aplicación y conceptualización del TPACK en diferentes áreas del conocimiento, esto nos permite enriquecer la investigación con muchas experiencias desarrolladas en múltiples asignaturas.

\section{Análisis de Resultados}

A partir del análisis bibliométrico realizado mediante la herramienta de VosViewer, ponemos en evidencia los países más relevantes que se encuentran investigando sobre el TPACK y su aplicación. En la figura 3 se muestra en escala de colores desde el violeta al amarillo los países que tienen las investigaciones más recientes entre los años 2018 y 2019 y como están relacionados entre sí.

\section{Figura 3}

Redes de investigaciones en TPACK entre países

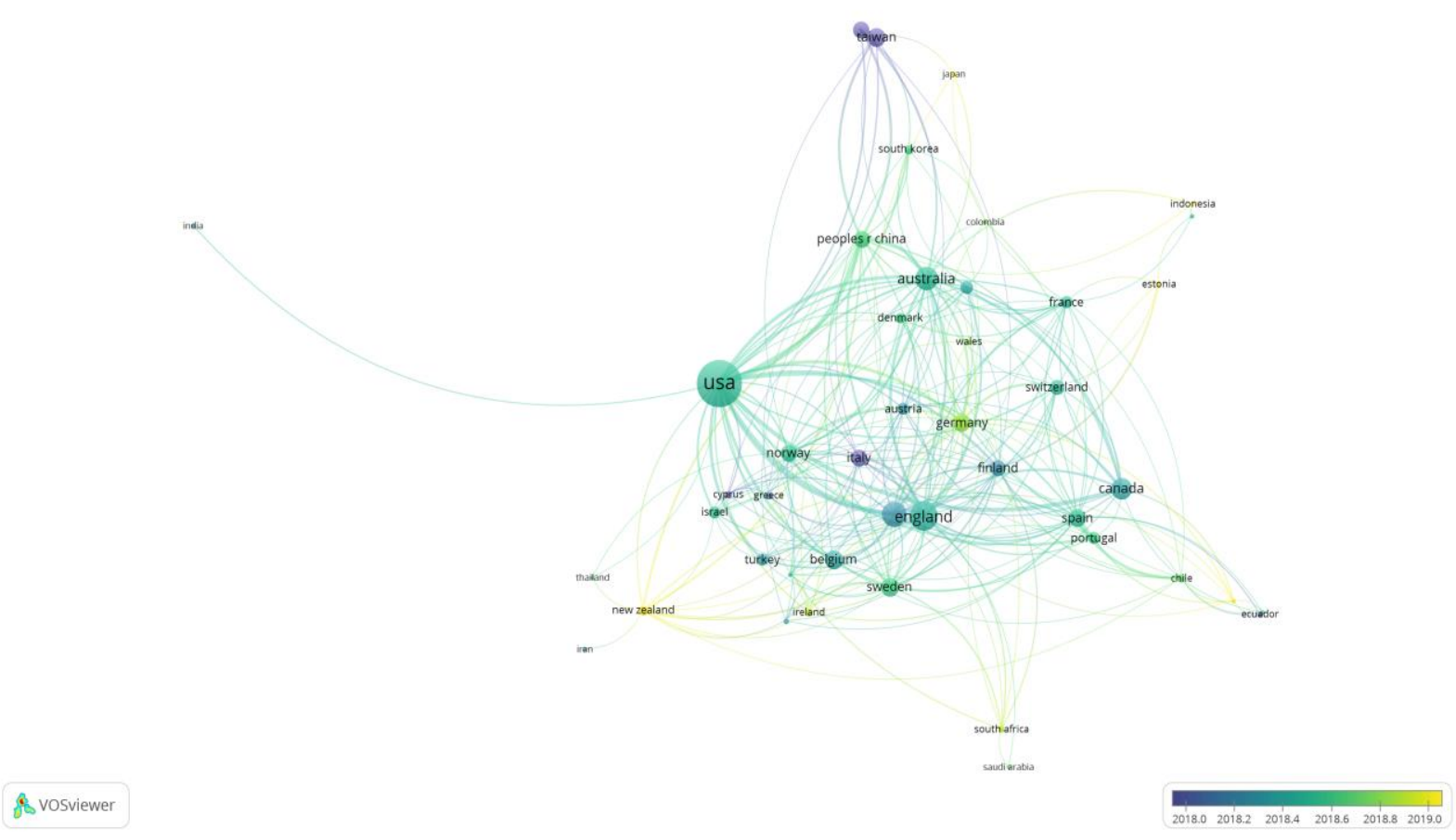


Entre los países que más resaltan por el número de estudios realizado se encuentra Estados Unidos, seguido de Australia, Alemania, Inglaterra y China. En el caso de los países latinoamericanos podemos observar que Chile está a la cabeza, seguido de Brasil, México y Colombia. Ecuador se encuentra en la cola con 5 artículos $^{1}$ publicados que abordan el TPACK. Esta gráfica nos permite dar razón fácilmente de los países potencia en cuanto a artículos referentes a TPACK y como están generando redes de estudio con otros países, así pues, esta figura nos permite acudir puntualmente a la búsqueda de artículos en esos países para realizar futuras investigaciones concernientes a TPACK. Esto da a notar que la producción científica más relevante y actualizada se encuentra en las grandes potencias, no así en los países de habla hispana a los que tradicionalmente solemos acudir por la facilidad del idioma.

De igual forma, hemos visto relevante identificar o conocer las universidades o instituciones que están abordando el tema y como estas entrelazan redes con otras universidades. Es lo que podemos observar en la figura 4. Sin embargo, aquí logramos identificar que el número de redes es inferior a las redes por países, es decir, las investigaciones se están haciendo con muy poca colaboración entre universidades.

\section{Figura 4}

Redes de investigaciones en TPACK entre universidades

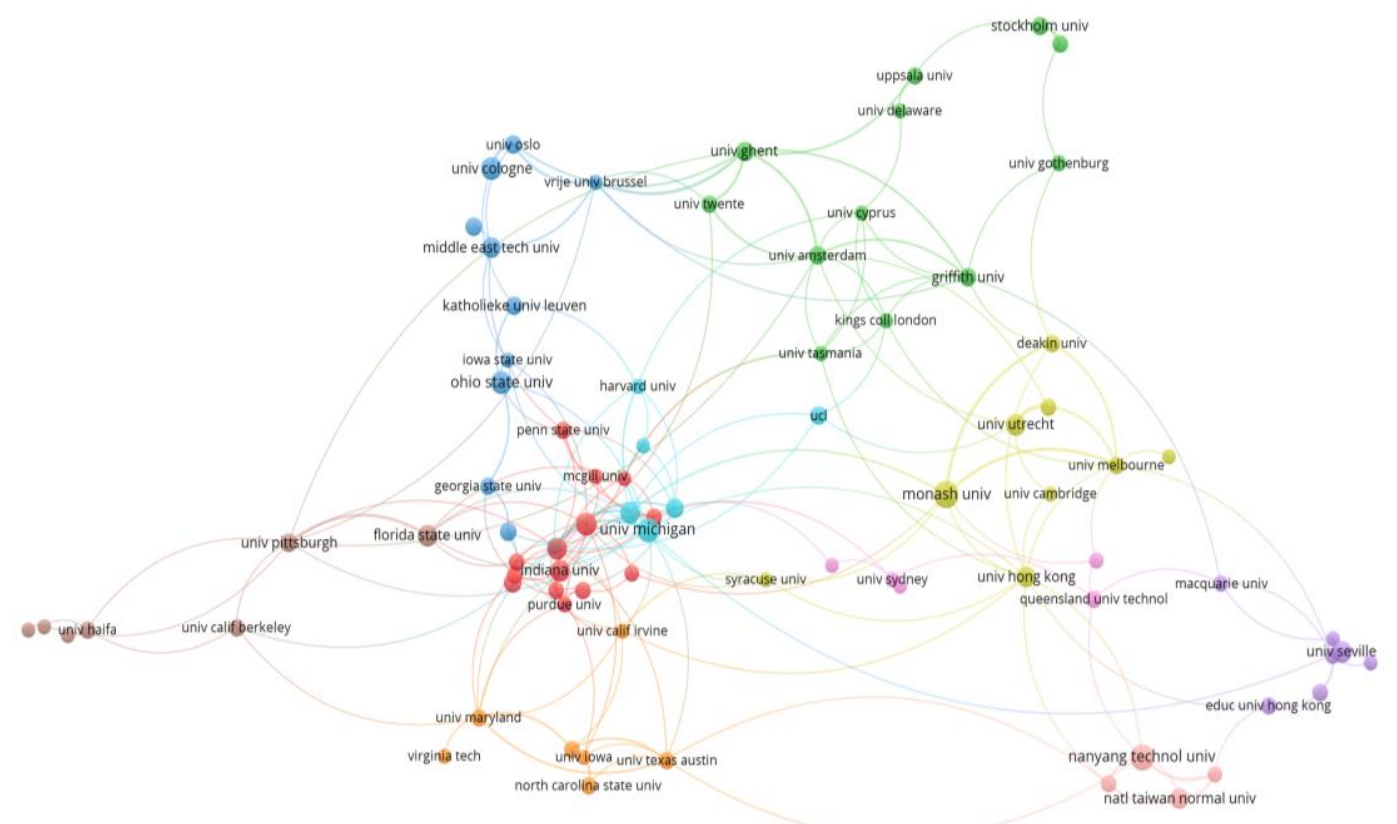

A vosviewer

Así mismo, a raíz del mismo análisis efectuado con la herramienta VosViewer hemos logrado identificar a los autores más relevantes que han venido realizado investigaciones sobre el

\footnotetext{
${ }^{1}$ Es el número de artículos hallados en la base de datos Web of Science. 
TPACK, en la tabla a continuación se detalla a estos autores y su impacto en las bases de datos de Web of Science y Scopus.

Tabla 2

Autores más relevantes sobre TPACK en las bases de datos más importantes

\begin{tabular}{lcclcc}
\hline \multicolumn{1}{c}{ Autores } & $\begin{array}{c}\text { Web of Science } \\
\text { Documentos }\end{array}$ & Citas & \multicolumn{1}{c}{ Autores } & $\begin{array}{c}\text { Scopus } \\
\text { Documentos }\end{array}$ & Citas \\
\hline Chai, C. $S$. & 20 & 556 & Tondeur $J$. & 22 & 716 \\
Koh, $J$. & 12 & 449 & Voogt J. & 18 & 355 \\
Tsai, C. & 7 & 274 & Siddiq F. & 9 & 355 \\
Tondeur, $J$. & 11 & 274 & Scherer . & 8 & 355 \\
Voogt, $J$. & 5 & 131 & Van Braak J. & 7 & 308 \\
Valtonen, $T$. & 6 & 73 & Chai C. S. & 28 & 234 \\
Koenig, $J$. & 15 & 72 & Fisser P. & 8 & 149 \\
Angeli, $C$. & 5 & 71 & Prestridge S. & 8 & 148 \\
Baran, $E$. & 5 & 47 & Mirsha P. & 7 & 122 \\
Depaepe, $F$. & 7 & 47 & Baran E. & 11 & 120 \\
\hline
\end{tabular}

Fuente: Web of Science y Scopus.

Entre los autores más importantes, se identificó a Chai, C. S., Koh, J., Tondeur, J., Tsai, C. y Voogt J. La producción científica de estos 5 autores hallada en Web of Science y Scopus suman un total de 95 artículos y un total de 2755 citas entre los años 2016 y 2020.

Además, a partir de la matriz propuesta, en la cual analizamos detalladamente 60 artículos científicos, se ha logrado evidenciar a los artículos científicos con mayor impacto. En la tabla 3 , damos cuenta de los 3 artículos de mayor impacto que se han encontrado.

\section{Tabla 3}

Artículos de mayor impacto

\begin{tabular}{|c|c|c|c|c|}
\hline Título & Autor/es & Citas & 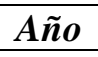 & Rank \\
\hline $\begin{array}{l}\text { Those who understand: Knowledge } \\
\text { growth in teaching }\end{array}$ & Shulman, Lee S. & 27917 & 1986 & $\begin{array}{l}\text { JCR Q1 } \\
\text { SJR Q1 }\end{array}$ \\
\hline $\begin{array}{l}\text { Knowledge and Teaching - } \\
\text { Foundations of the New Reform }\end{array}$ & Shulman, Lee S. & 23995 & 1987 & $\begin{array}{l}\text { JCR Q2 } \\
\text { SJR Q1 }\end{array}$ \\
\hline $\begin{array}{l}\text { Technological pedagogical content } \\
\text { knowledge: A framework for teacher } \\
\text { knowledge }\end{array}$ & $\begin{array}{l}\text { Mishra, Punya \& } \\
\text { Koehler, M. J. }\end{array}$ & 10671 & 2006 & $\begin{array}{l}\text { JCR Q4 } \\
\text { SJR Q3 }\end{array}$ \\
\hline
\end{tabular}

Así pues, dentro de este universo de autores que están abordando el TPACK, se ha visto que uno de los problemas más frecuentes es la escasa familiaridad con los recursos (tecnológicos) y la integración de estos en la cotidianidad educativa, es decir, existe un permanente divorcio entre las tres dimensiones base CK, PK y TK y sus derivados TCK, TPK y PCK. Se identificó que los docentes emplean cada dimensión como elementos separados, o a su vez presentan una relación dispar transformándose en dificultades al momento de integrar cada una de las dimensiones de 
TPACK (Arévalo Duarte et al., 2019) lo que puede significar falencias en el proceso de enseñanzaaprendizaje.

Por otro lado, la tecnología y el uso de la tecnología en la educación no pueden y deben considerarse como separados. En consecuencia, los docentes tienen un papel importante a la hora de integrar la tecnología en la educación, pues son los responsables directos de la misma (Demirok y Baglama, 2018). Para tratar de cerrar esas brechas entre cada uno de los constructos, sin duda la capacitación y del desarrollo de competencias toma un rol protagónico. De igual forma, creemos que es necesario motivar a los docentes, pero sobre todo empoderarlos.

Otro de los problemas encontrados es la capacitación o la adquisición de competencias digitales. Si bien es cierto, la tecnología es ineludible en nuestras vidas, esto no nos hace expertos en las mismas. Los autores revisados plantean la capacitación docente en competencias digitales. En este sentido, no solo basta con el conocimiento y manejo de las TIC, sino además hace falta saber diseñar o rediseñar los contenidos con el uso de TIC para que a su vez permitan adaptarse a las diferentes necesidades y contextos (Mohamad Nasri et al., 2020). No obstante, la capacitación (tradicional) de los docentes no brinda de experiencias necesarias para prepararlos para usar la tecnología de manera efectiva en sus aulas (Koehler et al., 2007), los cursos y talleres están quedando obsoletos, ya sea por la forma o la metodología de los mismos, por eso, más bien se busca un enfoque que permita a los docentes responder sus necesidades, compartir experiencias y sobre todo que contribuyan con la aplicación de los componentes del TPACK en situaciones prácticas (Krepf et al., 2018).

Se ha visto también, que, al generalizar el aprendizaje, muchos docentes pierden el interés porque ven alejados de su área de enseñanza, es decir, aprenden algo que no les va a servir. La capacitación docente en competencias digitales sin duda es uno de los pilares para la implementación de TPACK, sin esto, cualquier esfuerzo para que este modelo se aplique en las instituciones educativas es nulo. No basta que los docentes tengan el conocimiento del contenido y/o de la pedagogía. Hoy más que nunca hemos visto que el desarrollo del docente en las TIC se vuelve crucial mucho más en este tiempo de pandemia (Schildkamp et al., 2020) y sin duda en postpandemia.

Igualmente, en la revisión de la literatura científica, particularmente del 2020 se evidencia que el acceso a internet o la conectividad es un problema común que se avizoró en el contexto de la pandemia. La conectividad en geografías urbanas es más alta que en zonas rurales, sin embargo, el acceso a un ordenador parece ser uno de los problemas más comunes. En los datos recolectados desde Organización para la Cooperación y el Desarrollo Económicos (OECD) (2020a, 2020b, 2020c) hemos logrado ver como en los últimos 10 años (2009 al 2019) ha avanzado la curva ascendente, sin embargo, se ha visto momentos en donde la curva cayó ligeramente. En la figura 5 podemos evidenciar el nivel acceso a un ordenador en países como Chile, Colombia, Brasil, México y Costa Rica, están por debajo del 70\% (Organización para la Cooperación y el Desarrollo Económicos [OECD], 2020a). 


\section{Figura 5}

Acceso a un ordenador desde el hogar (OECD, 2020 $)$

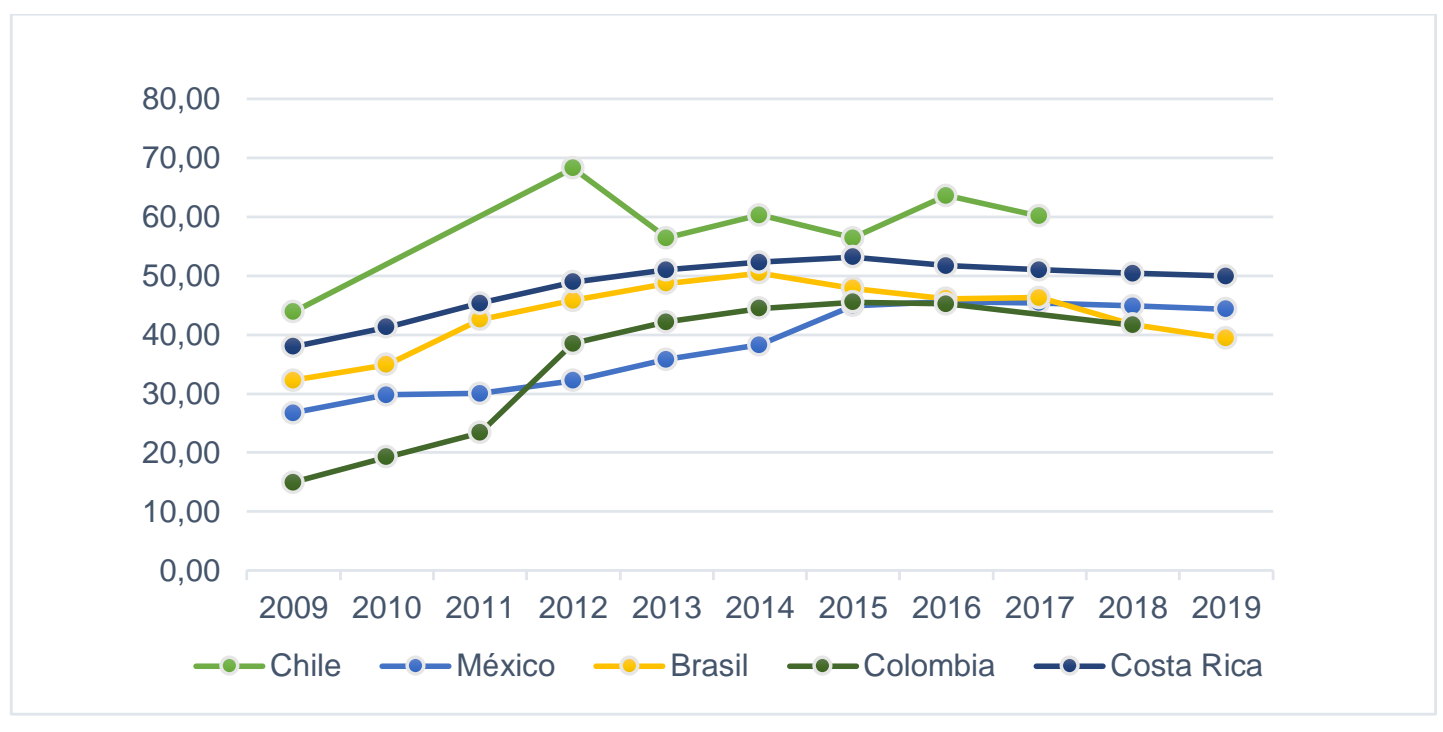

Vemos como países como Brasil, México, Colombia y Costa Rica están por debajo del $50 \%$, es decir que apenas la mitad de sus ciudadanos lograron acceder a un computador desde su hogar, sin duda estos datos se vuelven alarmantes, más aún cuando estamos atravesando una pandemia, en la que muchas personas han perdido sus empleos. Sin embargo, para el acceso a las TIC, no solo basta con disponer de un ordenador, también es necesario identificar el acceso al internet, es lo que vemos en la figura 6 con los mismos países antes vistos.

\section{Figura 6}

Acceso a internet (OECD, 2020c)

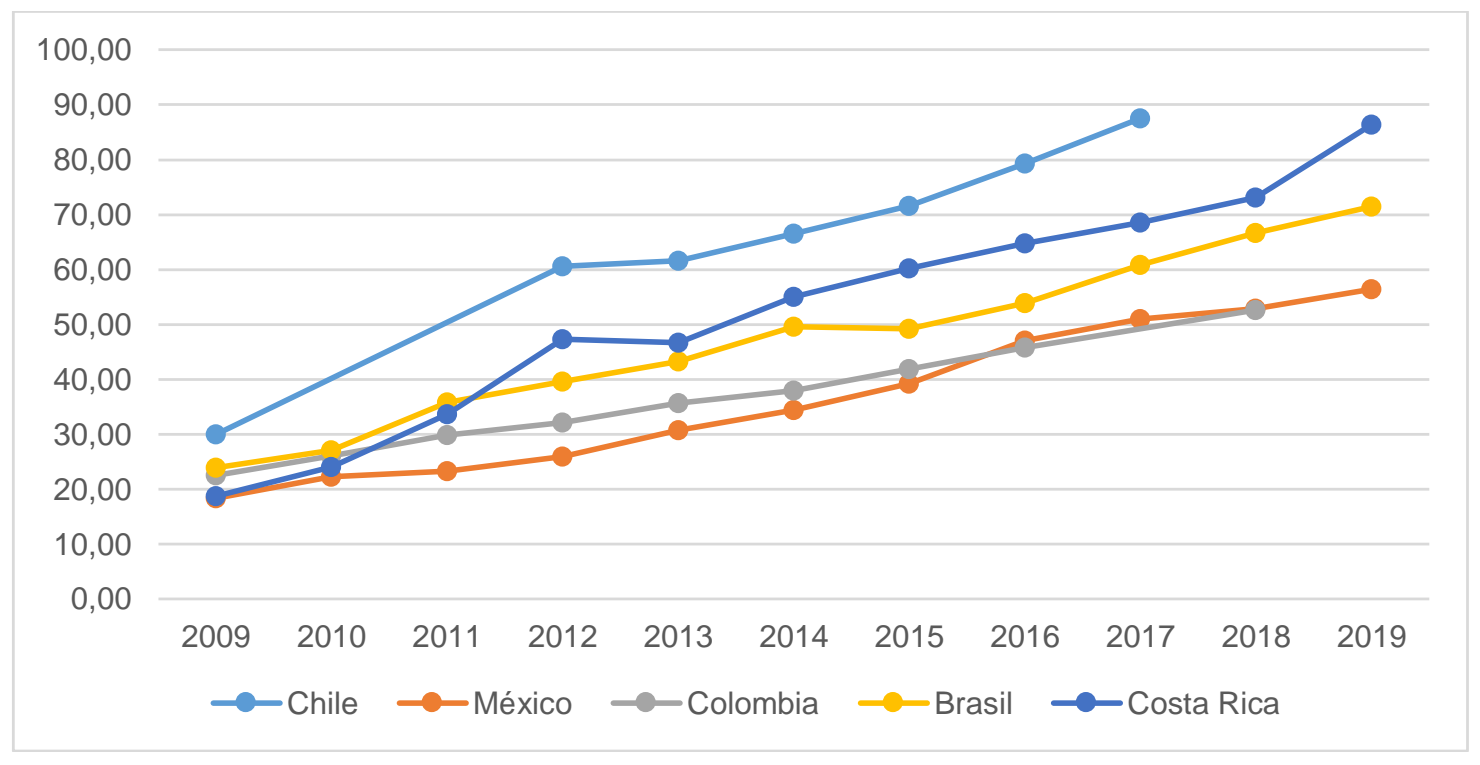

Esta obra se comparte bajo la licencia Creative Common Atribución-No Comercial 4.0 International (CC BY-NC 4.0) Revista de la Universidad Internacional del Ecuador. URL: https://www.uide.edu.ec/ 
Los datos reflejados en la figura 7, nos muestran cómo ha ido incrementando el acceso y sobre todo el uso de las TIC en los hogares de la región. Son cifras que están sobre el 50\% y que de alguna forma brindan cierto nivel de tranquilidad, no obstante, las brechas por el acceso a las TIC son muy amplias.

\section{Figura 7}

Acceso y uso de las TIC (OECD, 2020b)

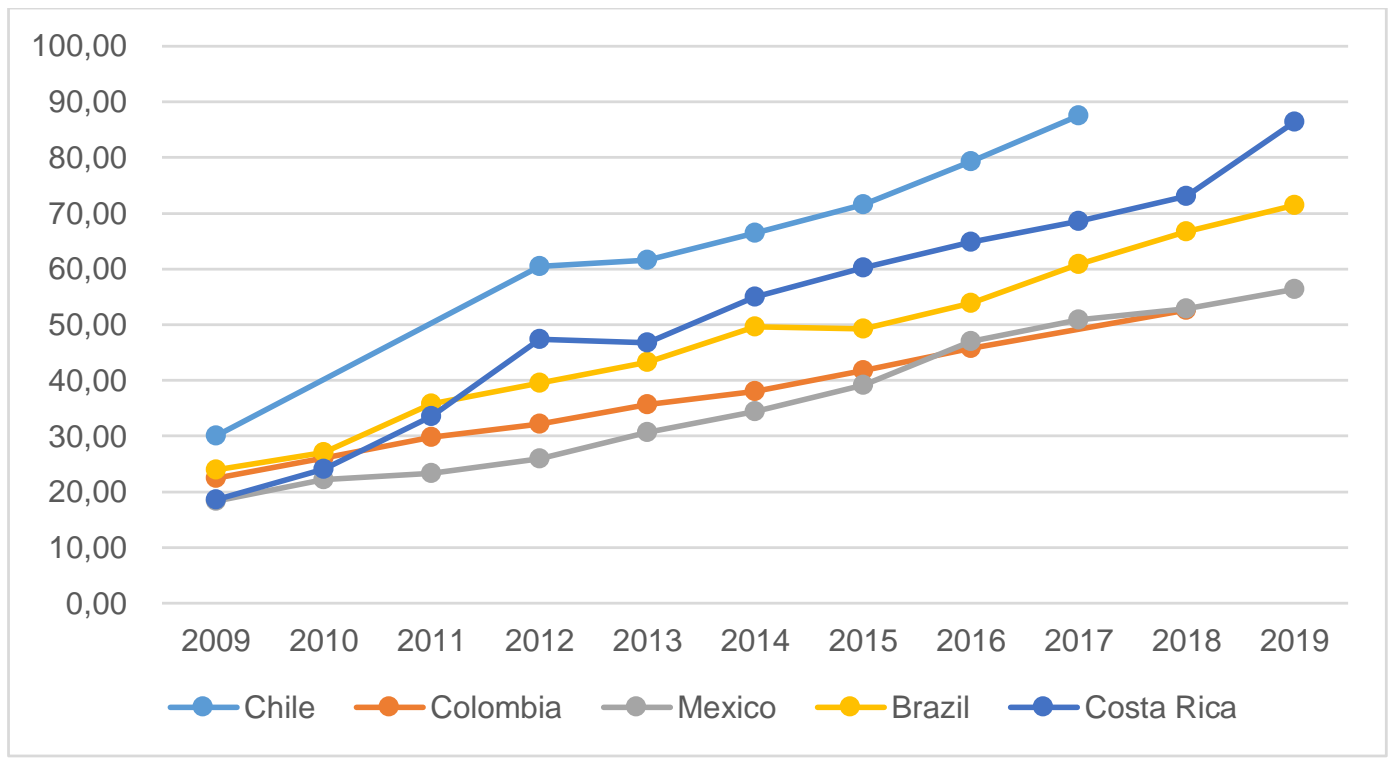

A partir de la figura 5, 6 y 7 podemos deducir varias cosas. Si bien es cierto hay un bajo nivel de personas que disponen de un ordenador en casa, muchas de esas personas podrían estar usando tecnologías emergentes como celulares o Tabletas, que además disponen de conexión a internet. Pues, como hemos visto en la figura 6 y 7 más de la media tienen acceso a internet y usan alguna TIC. En este sentido, vemos la importancia de las tecnologías emergentes que de alguna forma han permitido apaciguar las realidades y a la vez continuar la educación desde la virtualidad en tiempos de pandemia. Sin embargo, cabe notar que estas tecnologías emergentes no facilitan la visualización de documentos o videoconferencias por el tamaño de estos dispositivos.

Por otro lado, no se encontró datos en OECD sobre la situación del Ecuador, sin embargo, el Instituto Nacional de Estadísticas y Censo (INEC) en la Encuesta Multipropósito - TIC (2019a, 2019b) detalla que en 2019 en el Ecuador el 23,3\% tiene acceso a un computador de escritorio y el 28,5\% a un computador portátil. Por otro lado, sobre el acceso a internet, se evidencia que en las zonas rurales del Ecuador apenas el 21,6\% de la población tiene acceso a internet, mientras que en la zona urbana es el 56,1\%. No obstante, para diciembre de 2020, Internet World Stats (2021) señala que la penetración a internet para el Ecuador es del 80,2\% de la población, es decir son $14,340,687$ ecuatorianos. 
Algo que además llama nuestra atención son las creencias a la hora de implementar el TPACK. Es importante conocer las creencias de los profesores, pues suelen ser los más resistentes a la hora de implementar algo nuevo (Ekiz-Kiran y Boz, 2020). El costo, también es algo que llama nuestra atención, pues no es algo de consideración en la literatura revisada, pero vale notar que la formación docente y la infraestructura tecnológica requiere cierta inversión.

\section{Conclusiones}

Desarrollar estrategias para implementar el TPACK en los docentes requiere de un análisis integral, en el que debemos entender y conceptualizar lo que abarca el TPACK para posteriormente reconocer las necesidades y problemas, analizar los contextos o variables para que se pueda adaptar o (re)diseñar o redefinir el modelo según las necesidades. En todo este camino es importante disponer de formación continua y acompañamiento que de igual forma logre desarrollar las especificidades para cada asignatura (Xu et al., 2018), para cada nivel de estudios y sin duda para el contexto social y económico. Hemos visto que también es pertinente disponer de espacios de encuentro, en los que se pueda compartir experiencias o a su vez experimentar, es decir, espacios que permitan a los docentes conocer otras realidades para aprender de los aciertos y los errores al implementar TPACK. En este sentido la implementación y adaptación del TPACK se vuelve crucial para responder a las necesidades educativas, pues cada ambiente educativo es distinto por lo que no se puede aplicar TPACK como un recetario.

Si bien es cierto, las políticas no es un tema de preocupación en los artículos revisados, creemos que unas fuertes políticas educativas guiadas a la aplicación de modelos para mejorar la calidad educativa son necesarias. No se debe endosar todos los esfuerzos a los docentes y actuar de forma aislada, creemos que el trabajo colaborativo y en equipo entre toda la comunidad educativa es importante para fortalecer el proceso de enseñanza-aprendizaje y es justamente las entidades rectoras de la educación quienes deben dar lineamientos, motivar y facilitar la formación continua del docente, así como brindar, adecuar o facilitar el acceso de infraestructuras tecnológicas adecuadas. De igual forma, urge la incorporación de las TIC dentro del currículo educativo como herramientas no solo para (re)transmitir contenidos, sino además para facilitar la construcción, experimentación y finalmente ubicar al estudiante como actor y el centro del aprendizaje para así responder a las recientes teorías del aprendizaje.

Por otro lado, los estudios revisados analizan el TPACK desde la Autoevaluación del docente para cada una de las dimensiones de TPACK. En ese sentido, se han desarrollado varios instrumentos de autoevaluación siendo el más común el elaborado por Schmidt et al. (2009). Sin embargo, los instrumentos basados en la autoevaluación del docente para conocer el grado de aplicación de TPACK podría crear una imagen sesgada de la realidad, pues la autoevaluación puede resultar una sobreestimación o a su vez una subestimación de los conocimientos de cada dimensión. Así pues, es necesario empezar a triangular con otros actores como los mismos estudiantes para obtener resultados más fiables y apegados a la realidad.

Finalmente, dentro del modelo de TPACK, la implementación de TIC es categórico, en ese sentido se debe avanzar en el estudio del modelo SAMR, para de esta forma lograr ubicar el nivel de aplicación de TIC en los docentes. Con ello podremos evaluar una adecuada integración de 
tecnologías educativas que guarden pertinencia de acuerdo con los contextos para la enseñanzaaprendizaje.

\section{Referencias bibliográficas}

Angeli, C., \& Valanides, N. (2009). Epistemological and methodological issues for the conceptualization, development, and assessment of ICT-TPCK: Advances in technological pedagogical content knowledge (TPCK). Computers and Education, 52(1), 154-168. https://doi.org/10.1016/j.compedu.2008.07.006

Arévalo Duarte, M. A., García García, M. Á., y Hernández Suárez, C. A. (2019). Competencias TIC de los docentes de matemáticas en el marco del modelo TPACK: Valoración desde la perspectiva de los estudiantes. Civilizar, 19(36), 115-132. https://doi.org/10.22518/usergioa/jour/ccsh/2019.1/a07

Bostancıoğlu, A., \& Handley, Z. (2018). Developing and validating a questionnaire for evaluating the EFL 'Total PACKage': Technological Pedagogical Content Knowledge (TPACK) for English as a Foreign Language (EFL). Computer Assisted Language Learning, 31(5-6), 572-598. https://doi.org/10.1080/09588221.2017.1422524

Cabero, J., y Barroso, J. (2016). Formación del profesorado en TIC: Una visión del modelo $\begin{array}{lllll}\text { TPACK. Cultura } & y & \text { Educacion, } & \text { 633-663), }\end{array}$ https://doi.org/10.1080/11356405.2016.1203526

Canbazoglu Bilici, S., Guzey, S. S., \& Yamak, H. (2016). Assessing pre-service science teachers' technological pedagogical content knowledge (TPACK) through observations and lesson plans. Research in Science and Technological Education, 34(2), 237-251. https://doi.org/10.1080/02635143.2016.1144050

Cenich, G., Araujo, S., y Santos, G. (2019). Conocimiento tecnológico pedagógico del contenido en la enseñanza de matemática en el ciclo superior de la escuela secundaria. Perfiles Educativos, 42(167), 53-67. https://doi.org/10.22201/iisue.24486167e.2019.167.59276

Chai, C. S., Koh, J. H. L., \& Tsai, C.-C. (2010). Facilitating Preservice Teachers' Development of Technological, Pedagogical, and Content Knowledge (TPACK). Educational Technology \& Society, 13(4), 63-73. www.jstor.org/stable/jeductechsoci.13.4.63.

Cheng, K. H. (2017). A survey of native language teachers' technological pedagogical and content knowledge (TPACK) in Taiwan. Computer Assisted Language Learning, 30(7), 692-708. https://doi.org/10.1080/09588221.2017.1349805

Demirok, M. S., \& Baglama, B. (2018). Examining technological and pedagogical content knowledge of special education teachers based on various variables. TEM Journal, 7(3), 507-512. https://doi.org/10.18421/TEM73-06

Depaepe, F., Verschaffel, L., \& Star, J. (2020). Expertise in developing students' expertise in mathematics: Bridging teachers' professional knowledge and instructional quality. ZDM Mathematics Education, 52(2), 179-192. https://doi.org/10.1007/s11858-020-01148-8

Ekiz-Kiran, B., \& Boz, Y. (2020). Interactions between the science teaching orientations and components of pedagogical content knowledge of in-service chemistry teachers. Chemistry Education Research and Practice, 21(1), 95-112. https://doi.org/10.1039/c9rp00092e

Getenet, S. T. (2017). Adapting technological pedagogical content knowledge framework to teach 
mathematics. Education and Information Technologies, 22(5), 2629-2644. https://doi.org/10.1007/s10639-016-9566-x

Hsu, L. W., \& Chen, Y. J. (2019). Examining teachers' technological pedagogical and content knowledge in the era of cloud pedagogy. South African Journal of Education, 39(December), 1-13. https://doi.org/10.15700/saje.v39ns2a1572

Instituto Nacional de Estadisticas y Censo INEC. (2019a). Indicadores de tecnología de la información y comunicación. En Encuesta de Seguimiento al Plan Nacional de Desarrollo. https://bit.ly/3jw7GKv

Instituto Nacional de Estadisticas y Censo INEC. (2019b). Tecnologías de la Información y Comunicación. Encuesta multiporpósito - TIC 2019. En Inec. https://www.ecuadorencifras.gob.ec/tecnologias-de-la-informacion-y-comunicacion-tic/

Internet World Stats. (2021). Usage and Population Statistics. https://www.internetworldstats.com/south.htm\#ec

Koehler, M. J., Mishra, P., \& Yahya, K. (2007). Tracing the development of teacher knowledge in a design seminar: Integrating content, pedagogy and technology. Computers and Education, 49(3), 740-762. https://doi.org/10.1016/j.compedu.2005.11.012

Koh, J. H. L., Chai, C. S., \& Lim, W. Y. (2017). Teacher Professional Development for TPACK21CL: Effects on Teacher ICT Integration and Student Outcomes. Journal of Educational Computing Research, 55(2), 172-196. https://doi.org/10.1177/0735633116656848

König, J., Jäger-Biela, D. J., \& Glutsch, N. (2020). Adapting to online teaching during COVID-19 school closure: teacher education and teacher competence effects among early career teachers in Germany. European Journal of Teacher Education, 43(4), 608-622. https://doi.org/10.1080/02619768.2020.1809650

Krepf, M., Plöger, W., Scholl, D., \& Seifert, A. (2018). Pedagogical content knowledge of experts and novices - what knowledge do they activate when analyzing science lessons? Journal of Research in Science Teaching, 55(1), 44-67. https://doi.org/10.1002/tea.21410

Ku, C. J., Loh, W. L. L., Lin, K. Y., \& John Williams, P. (2020). Development of an instrument for exploring preservice technology teachers' maker-based technological pedagogical content knowledge. British Journal of Educational Technology, 0(0), 1-17. https://doi.org/10.1111/bjet.13039

Ling Koh, J. H., Chai, C. S., \& Tay, L. Y. (2014). TPACK-in-Action: Unpacking the contextual influences of teachers' construction of technological pedagogical content knowledge (TPACK). Computers $\quad$ and 20-29. https://doi.org/10.1016/j.compedu.2014.04.022

Mishra, P., \& Koehler, M. J. (2006). Technological Pedagogical Content Knowledge: A Framework for Teacher Knowledge. Teachers College Record, 108(6), 1017-1054. https://doi.org/10.1111/j.1467-9620.2006.00684.x

Mohamad Nasri, N., Husnin, H., Mahmud, S. N. D., \& Halim, L. (2020). Mitigating the COVID19 pandemic: a snapshot from Malaysia into the coping strategies for pre-service teachers' education. Journal of Education for Teaching, 00(00), 1-8. https://doi.org/10.1080/02607476.2020.1802582

Morán Peña, F. L., Morán Peña, F. E., y Albán Sánchez, J. D. (2017). Formación del docente y su adaptación al modelo TPACK. Revista Ciencias Pedagógicas E Innovación, 5(1), 51-60. 
https://doi.org/10.26423/rcpi.v5i1.154

OECD. (2020a). Access to computers from home (indicator). https://doi.org/10.1787/a70b8a9f-en

OECD. (2020b). ICT Access and Usage by Households and Individuals. OECD Telecommunications and Internet Statistics (database). https://doi.org/10.1787/b9823565en

OECD. (2020c). Internet Access (indicator). https://doi.org/10.1787/69c2b997-en

Ortega-Sánchez, D., \& Gómez-Trigueros, I. M. (2019). Didactics of historical-cultural heritage QR codes and the TPACK model: An analytic revision of three classroom experiences in Spanish higher education contexts. Education Sciences, 9(2), 1-10. https://doi.org/10.3390/educsci9020117

Rap, S., Feldman-Maggor, Y., Aviran, E., Shvarts-Serebro, I., Easa, E., Yonai, E., Waldman, R., \& Blonder, R. (2020). An Applied Research-Based Approach to Support Chemistry Teachers during the COVID-19 Pandemic. Journal of Chemical Education, 97(9), 32783284. https://doi.org/10.1021/acs.jchemed.0c00687

Schildkamp, K., Wopereis, I., Kat-De Jong, M., Peet, A., \& Hoetjes, Ij. (2020). Building blocks of instructor professional development for innovative ICT use during a pandemic. Journal of Professional Capital and Community, 5(3-4), 281-293. https://doi.org/10.1108/JPCC06-2020-0034

Schmidt, D. A., Baran, E., Thompson, A. D., Mishra, P., Koehler, M. J., \& Shin, T. S. (2009). Technological pedagogical content knowledge (Track): The development and validation of an assessment instrument for preservice teachers. Journal of Research on Technology in Education, 42(2), 123-149. https://doi.org/10.1080/15391523.2009.10782544

Shulman, L. S. (1986). Those Who Understand: Knowledge Growth in Teaching. Educational Researcher, 15(2), 4-14. https://doi.org/10.2307/1175860

Shulman, L. S. (1987). Knowledge and Teaching:Foundations of the New Reform. Harvard Educational Review, 57(1), 1-23. https://doi.org/10.17763/haer.57.1.j463w79r56455411

Sumba Nacipucha, N., Cueva Estrada, J. M., Conde Lorenzo, E., y Mármol Castillo, M. (2020). Enseñanza superior en el Ecuador en tiempos de COVID 19 en el marco del modelo TPACK. Revista San Gregorio, 43, 171-186. https://doi.org/10.36097/rsan.v1i43.1524

Sutton, K. K., \& DeSantis, J. (2017). Beyond change blindness: embracing the technology revolution in higher education. Innovations in Education and Teaching International, 54(3), 223-228. https://doi.org/10.1080/14703297.2016.1174592

Thompson, A. D., \& Mishra, P. (2007). Breaking News: TPCK becomes TPACK! Journal of Computing in Teacher Education, 24(2), 38-64. https://doi.org/10.1080/10402454.2007.10784583

Tondeur, J., Sinnaeve, I., van Houtte, M., \& van Braak, J. (2011). Ict as cultural capital: The relationship between socioeconomic status and the computer-use profile of young people. New Media and Society, 13(1), 151-168. https://doi.org/10.1177/1461444810369245

Valtonen, T., Sointu, E., Kukkonen, J., Kontkanen, S., Lambert, M. C., \& Mäkitalo-Siegl, K. (2017). TPACK updated to measure pre-service teachers' twenty-first century skills. Australasian Journal of Educational Technology, 33(3), 15-31. https://doi.org/10.14742/ajet.3518

Voogt, J., Fisser, P., Pareja Roblin, N., Tondeur, J., \& van Braak, J. (2013). Technological 
pedagogical content knowledge - A review of the literature. Journal of Computer Assisted Learning, 29(2), 109-121. https://doi.org/10.1111/j.1365-2729.2012.00487.x

Xu, S., Zhu, S., \& Tang, M. (2018). A Research on the Present Situation and Strategies of PreService Teachers' TPACK Competence. Proceedings - 9th International Conference on Information Technology in Medicine and Education, ITME 2018, 353-356. https://doi.org/10.1109/ITME.2018.00085 